\title{
Generation and structural characterization of aluminum cyanoacetylide
}

\author{
Carlos Cabezas, ${ }^{1}$ Carmen Barrientos, ${ }^{2}$ Antonio Largo, ${ }^{2, a)}$ Jean-Claude Guillemin, ${ }^{3}$ \\ José Cernicharo, ${ }^{4}$ Isabel Peña, ${ }^{1}$ and José L. Alonso ${ }^{1, a)}$ \\ ${ }^{1}$ Grupo de Espectroscopia Molecular (GEM), Edificio Quifima, \\ Laboratorios de Espectroscopia y Bioespectroscopia, Unidad Asociada CSIC, Parque Científico Uva, \\ Universidad de Valladolid, Paseo de Belén 5, 47011 Valladolid, Spain \\ ${ }^{2}$ Departamento de Química Física y Química Inorgánica, Facultad de Ciencias, Universidad de Valladolid, \\ Campus Miguel Delibes, Paseo de Belén 7, 47011 Valladolid, Spain \\ ${ }^{3}$ Institut des Sciences Chimiques de Rennes, École Nationale Supérieure de Chimie de Rennes, CNRS, \\ UMR 6226, 11 Allée de Beaulieu, CS 50837, 35708 Rennes Cedex 7, France \\ ${ }^{4}$ Group of Molecular Astrophysics, ICMM C/Sor Juana Ines de la Cruz, N3 Cantoblanco, \\ 28049 Madrid, Spain
}

(Received 30 May 2014; accepted 19 August 2014; published online 8 September 2014)

\begin{abstract}
Combined spectroscopy measurements and theoretical calculations bring to light a first investigation of a metallic cyanoacetylide, $\mathrm{AlC}_{3} \mathrm{~N}$, using laser ablation molecular beam Fourier transform microwave spectroscopy. This molecule was synthesized in a supersonic expansion by the reaction of aluminum vapour with $\mathrm{C}_{3} \mathrm{~N}$, produced from solid aluminum rods and $\mathrm{BrCCCN}$ in a newly constructed ablation-heating nozzle device. A set of accurate rotational and ${ }^{27} \mathrm{Al}$ and ${ }^{14} \mathrm{~N}$ nuclear quadrupole coupling constants have been determined from the analysis of the rotational spectrum and compared with those predicted in a high-level ab initio study, conducting to the assignment of the observed species to linear AlCCCN. We have searched for this species towards the carbon-rich evolved star IRC +10216 but only an upper limit to its abundance has been obtained. ( 2014 AIP Publishing LLC. [http://dx.doi.org/10.1063/1.4894501]
\end{abstract}

\section{INTRODUCTION}

The nature of the metal-carbon chemical bonding in organometallic species has not been extensively explored, at least from a spectroscopic point of view, and it remains a topic of considerable interest. The experimentally determined molecular parameters of these molecules offer a unique opportunity to examine the competition between ionic and covalent bonding. Since these compounds play a wide range of roles in chemistry, establishing their structures provides a deeper understanding in, among others, catalytic processes and growth mechanisms of nanomaterials. Metal-bearing species are also of interest in astrochemistry. They were detected in space for the first time in the circumstellar envelope of the carbon-rich evolved star IRC $+10216 .{ }^{1}$ Since then, the number of metal-bearing molecules detected in the interstellar medium has increased significantly. So far, the list of interstellar molecules includes species with $\mathrm{Na}, \mathrm{K}, \mathrm{Mg}$, $\mathrm{Al}$, and even transition metals such as Fe or Ti. In particular, among refractory species, aluminum-bearing molecules are predicted to be especially abundant according to recent studies. ${ }^{2}$ Known interstellar aluminum-bearing molecules include halides $\left(\mathrm{AlF}^{1,3} \mathrm{AlCl}^{1}\right)$, the oxide and hydroxide species $\left(\mathrm{AlO},{ }^{4} \mathrm{AlOH}^{5}\right)$, as well as aluminum isocyanide, AlNC. ${ }^{6}$ Other metal-containing cyanides and isocyanides have been observed in the interstellar medium. The list includes $\mathrm{NaCN},{ }^{7}$ $\mathrm{KCN},{ }^{8} \mathrm{MgNC},{ }^{9} \mathrm{MgCN},{ }^{10}$ and FeCN. ${ }^{11}$ Silicon cyanide and

\footnotetext{
a) Authors to whom correspondence should be addressed. Electronic addresses: alargo@qf.uva.es, Telephone: +34 983423482 and jlalonso@qf.uva.es, Telephone: +34 983186345.
}

isocyanide have been also detected, ${ }^{12,13}$ and very recently the observation of a related magnesium molecule, $\mathrm{HMgNC}$, was reported. ${ }^{14}$

The presence of a relatively wide variety of cyanides and isocyanides in space opens the possibility for the detection of longer carbon chains containing metals similar to the cyanopolyynes family: $\mathrm{HC}_{3} \mathrm{~N}, \mathrm{HC}_{5} \mathrm{~N}, \mathrm{HC}_{7} \mathrm{~N}, \mathrm{HC}_{9} \mathrm{~N}$, and $\mathrm{HC}_{11} \mathrm{~N}$. In fact, $\mathrm{HC}_{11} \mathrm{~N}$ is the longest linear molecule observed in space ${ }^{15}$ so far. Analogue species containing metals could be synthesized through similar reaction schemes as those proposed for the family of cyanopolyynes. ${ }^{16}$ However, the possible detection of metal chains is hindered by the lack of spectroscopic information on these compounds. To the best of our knowledge none of these potential species have been characterized in laboratory.

In the present work we generated and characterized the first member of the Metal- $\mathrm{C}_{3} \mathrm{~N}$ series, containing aluminum and with formula $\mathrm{AlC}_{3} \mathrm{~N}$. Laser ablation Fourier transform microwave spectroscopy (LA-MB-FTMW) ${ }^{17}$ has been used to generate $\mathrm{AlC}_{3} \mathrm{~N}$ and characterize its rotational spectrum across the $2-12 \mathrm{GHz}$ frequency region. To conduct this study, a nozzle which combines a heating-reservoir with laser ablation capabilities has been developed, in order to employ solid precursors to generate the desired species. We have also carried out a complete computational study to guide the identification of the generated species. Finally, a search for the detected species has been performed towards IRC +10216 but only upper limits have been derived. Experimental and computational strategies are described in Secs. II and III. 


\section{EXPERIMENTAL}

\section{A. LA-MB-FTMW spectroscopy}

The $\mathrm{AlC}_{3} \mathrm{~N}$ measurements were carried out using a new LA-MB-FTMW spectrometer ${ }^{18}$ constructed at the University of Valladolid specially designed to maximize performances at low frequency ranges (from 2 to $12 \mathrm{GHz}$ ). It has been recently employed in the studies of large biomolecules. ${ }^{18,19}$ In this investigation, our conventional ablation nozzle holder ${ }^{17}$ has been modified by addition of a home-made heatable reservoir extension, placed between the valve and the laser ablation nozzle. The reservoir can hold liquid and/or solid compounds that can be used as precursors of metal bearing species. This new device is accommodated in the backside of the fixed mirror, aligned parallel relative to the optical axis of the resonator.

Solid samples of BrCCCN were synthesized by bromination of cyanoacetylene as previously reported ${ }^{20}(60 \mathrm{mmol}$ $(7.8 \mathrm{~g})$ were obtained in one run starting from $0.1 \mathrm{~mol}$ of $\mathrm{HC}_{3} \mathrm{~N}$ and an aqueous $\mathrm{Br}^{-}-\mathrm{Br}_{2} / \mathrm{KOH}$ solution). The precursor was placed in the heatable reservoir to produce a jet expansion with enough concentration of cyanoacetylide. Hence, $\mathrm{AlC}_{3} \mathrm{~N}$ was created by laser ablation of aluminum rods in the throat of a pulsed supersonic expansion of Ne (10 bars stagnation pressure) and $\mathrm{BrCCCN}$. A pulsed picosecond $\mathrm{Nd}$ :YAG-laser $\left(\lambda=355 \mathrm{~nm}\right.$ and $10 \mathrm{~mJ}$ pulse $\left.{ }^{-1}\right)$ at a repetition rate of $2 \mathrm{~Hz}$ was used. A motorized micrometer rotates and translates the sample rod each laser pulse, so the laser hits a different point of the sample surface in successive pulses, minimizing the problem of shot-to-shot fluctuation in the amount of the desorbed material.

Briefly, the sequence of an experimental cycle starts with a gas pulse of the mixing carrier gas (typically $450 \mu \mathrm{s}$ ). After an adequate delay, a laser pulse hits the metal rod, vaporizing the solid and producing plasma which trigger the chemical reactions in the precursor mixture. ${ }^{21}$ Immediately, the resulting products are supersonically expanded between the two mirrors of the Fabry-Pérot resonator and then a microwave pulse $(0.3 \mu \mathrm{s})$ is applied, producing the macroscopic polarization of the species in the jet. Once the excitation ceases, molecular relaxation gives rise to a transient emission signal (free induction decay) at microwave frequencies, which is captured in the time domain. Its Fourier transformation to the frequency domain yields the rotational transitions that appear as Doppler doublets, because the supersonic jet travels parallel to the resonator axis. The molecular rest frequencies are calculated as the arithmetic mean of the Doppler doublets and are obtained with accuracy better than $3 \mathrm{kHz}$. Typically 100-200 pulses were accumulated to achieve an adequate signal-to-noise ratio.

\section{B. Computational methods}

We have carried out a survey of the $\mathrm{AlC}_{3} \mathrm{~N}$ potential hypersurface in order to characterize possible stable isomers. We are only aware of a previous theoretical study of $\mathrm{AlC}_{3} \mathrm{~N}$ isomers by Petrie, ${ }^{22}$ where just two isomers were considered. For our purpose we employed the second-order MØller-Plesset $(\mathrm{MP} 2)^{23}$ level of theory with Dunning's correlated consistent triple-zeta basis sets augmented with diffuse and polarization functions, usually denoted as aug-cc-pVTZ. ${ }^{24}$ Harmonic vibrational calculations were carried out for the stationary points obtained through this exploration in order to confirm that they correspond to true minima. For the more significant species characterized through this procedure we carried out subsequent CCSD(T) calculations ${ }^{25}$ (coupled cluster with singles and doubles and a perturbative inclusion of triple excitations) with the aug-cc-pVTZ basis set.

In order to aid in the assignment of the rotational spectrum, reliable predictions for the rotational constant, as well as nuclear quadrupole coupling constants, are required. $\mathrm{AlC}_{3} \mathrm{~N}$ possesses two different nuclei with quadrupole moment, ${ }^{27} \mathrm{Al}$ $(I=5 / 2)$ and ${ }^{14} \mathrm{~N}(I=1)$, which interact with the electric field gradient created by the rest of the molecule at the nucleus, splitting into a very complex hyperfine pattern each rotational transition. For the geometrical parameters two different procedures have been employed, CCSD(T) optimizations with Dunning's quadruple-zeta basis set, aug-cc-pVQZ and a composite procedure have been applied. This type of schemes at the coupled-cluster level has been developed by Gauss et $a .^{26,27}$ A more affordable version of the method, in terms of computational demands, involving geometry optimizations at the MP2 level has been used by Barone et al. ${ }^{28}$ as an alternative to expensive coupled-cluster calculations, showing also good results. In this context, we have also adopted this MP2based composite method, to check its performance as an alternative to the expensive $\operatorname{CCSD}(\mathrm{T}) / \mathrm{aug}$-cc-pVQZ that we have also carried out. For a detailed description of the method we refer to the paper of Barone et al. ${ }^{28}$

Employing both theoretical procedures, CCSD(T)/augcc-pVQZ and the composite scheme, equilibrium spectroscopic parameters were obtained. In order to achieve an estimate of $\mathrm{B}_{0}$ rotational constant, vibration-rotation interaction constants were estimated using second-order perturbation theory at the MP2/aug-cc-pVTZ level. Aditionally, to aid in a possible experimental detection by IR spectroscopy, anharmonic vibrational frequencies and IR intensities have been predicted at that level of theory (Table S1 of the supplementary material ${ }^{45}$ ). Both Gaussian $09^{29}$ (MP2 geometries) and $\mathrm{CFOUR}^{30}$ (CCSD(T) geometries) program packages were employed in this study.

\section{RESULTS AND DISCUSSION}

After exploring the $\mathrm{AlC}_{3} \mathrm{~N}$ potential hypersurface four linear isomers were obtained, namely AlCCCN, AlCCNC, AlCNCC, and AlNCCC (Figure 1). We also considered cyclic structures with aluminum in the middle of the chain. Thus, following the imaginary normal modes the optimization ended up with either $\mathrm{CN}-\mathrm{Al}\left(\mathrm{C}_{2}\right)$ and $\mathrm{NC}-\mathrm{Al}\left(\mathrm{C}_{2}\right)$. These two structures have an $\mathrm{AlC}_{2}$ triangle subunit bonded to $\mathrm{CN}$ either through the nitrogen or the carbon atom, respectively. The relative energies of all these species, as well as their rotational constants and dipole moments obtained at the MP2/aug-ccpVTZ level, are summarized in Table I, and the equilibrium bond distances for these isomers obtained at the same level are provided in Figure 1. 

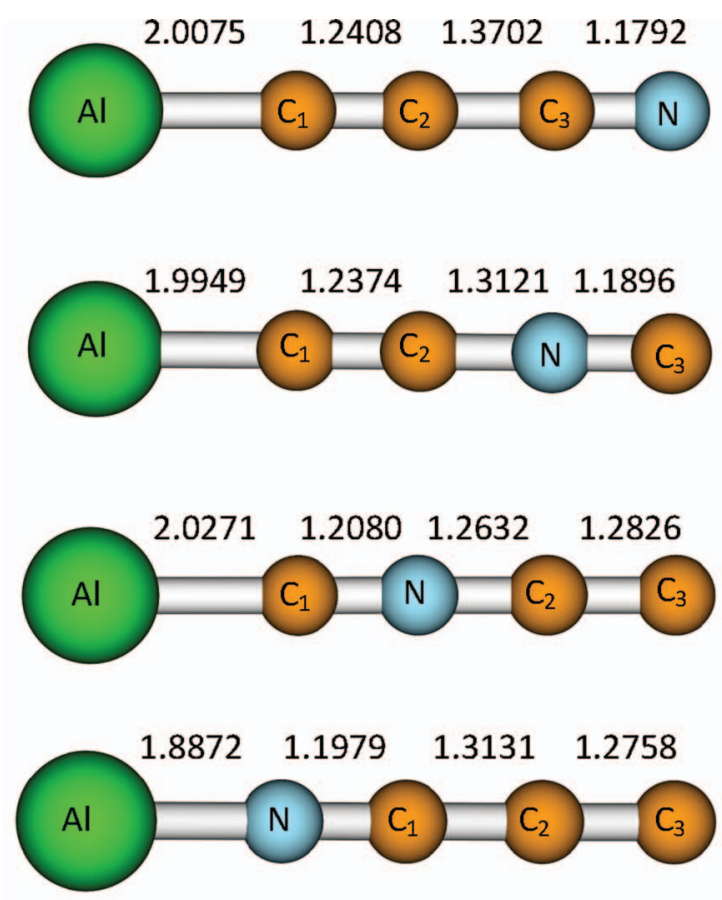

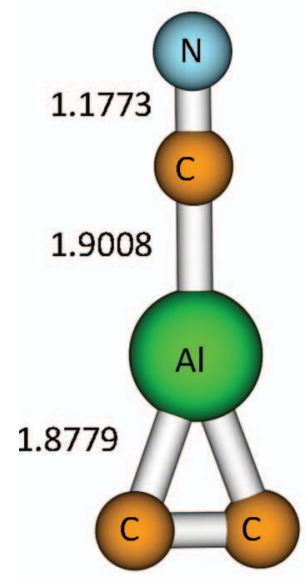

1.2862

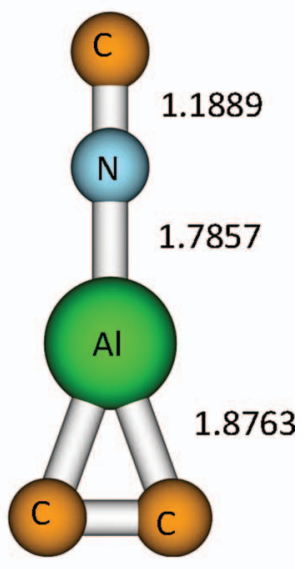

1.2867
FIG. 1. Equilibrium geometrical parameters for the different $\mathrm{AlC}_{3} \mathrm{~N}$ isomers obtained at the MP2/aug-cc-pVTZ level. Distances are given in Ångstroms.

TABLE I. Relative energies ( $\mathrm{kcal} / \mathrm{mol})$ of the different $\mathrm{AlC}_{3} \mathrm{~N}$ species obtained at the MP2 and CCSD(T) levels with the aug-cc-pVTZ basis set. Zero-point vibrational energy (ZPVE) corrections are taken into account at the MP2/aug-cc-pVTZ level. Equilibrium rotational constants $(\mathrm{MHz})$ and dipole moments (Debye) are estimated at the MP2/aug-cc-pVTZ level and CCSD/aug-cc-pVTZ levels, respectively.

\begin{tabular}{lcccccc}
\hline \hline Species & $\Delta \mathrm{E}(\mathrm{MP} 2)$ & $\begin{array}{c}\Delta \mathrm{E} \\
(\mathrm{CCSD}(\mathrm{T}))\end{array}$ & $\mathrm{A}$ & $\mathrm{B}$ & $\mathrm{C}$ & $\mu$ \\
\hline AlCCCN & 0.0 & 0.0 & & 1317.0 & & 3.908 \\
AlCCNC & 30.66 & 26.04 & & 1395.8 & & 3.102 \\
AlCNCC & 65.18 & 66.23 & & 1419.7 & & 7.379 \\
AlNCCC & 24.71 & 22.07 & & 1480.9 & & 7.457 \\
$\mathrm{CN}-\mathrm{Al}\left(\mathrm{C}_{2}\right)$ & 29.1 & 25.58 & 50875.0 & 2286.9 & 2188.5 & 1.620 \\
$\mathrm{NC}-\mathrm{Al}\left(\mathrm{C}_{2}\right)$ & 25.4 & 25.68 & 50914.0 & 2068.1 & 1987.4 & 1.595 \\
\hline \hline
\end{tabular}
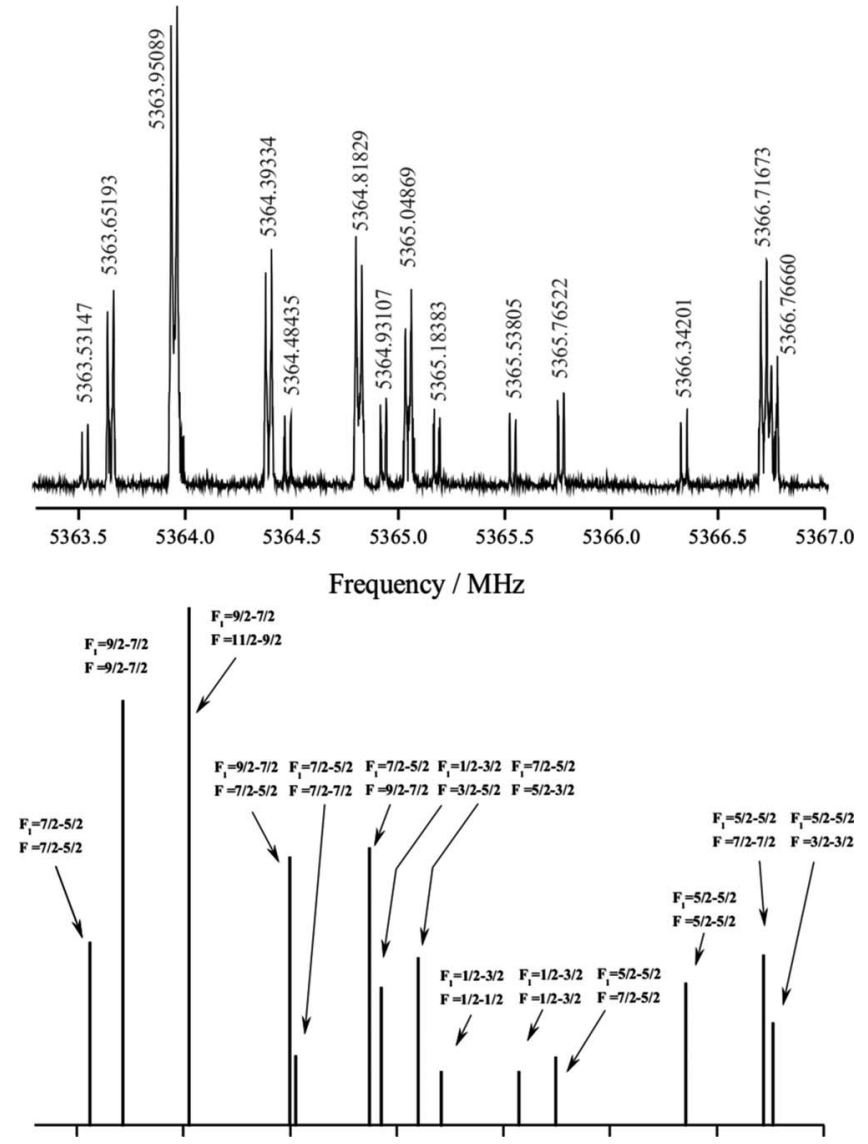

FIG. 2. (Upper panel) Central section of the $J=2-1$ rotational transition of $\mathrm{AlC}_{3} \mathrm{~N}$ near $5.4 \mathrm{GHz}$. The nuclear quadrupole coupling hyperfine structure arising from both ${ }^{27} \mathrm{Al}(\mathrm{I}=5 / 2)$ and ${ }^{14} \mathrm{~N}(\mathrm{I}=1)$ nuclei is clearly resolved. The coaxial arrangement of the adiabatic expansion and the resonator axis produces an instrumental Doppler doubling. The resonances frequencies are calculated as the average of the two Doppler components. (Lower panel) Theoretical simulation of the nuclear quadrupole hyperfine structure for the $J=2-1$ rotational transition of AlCCCN isomer.

The energy results show that linear AlCCCN is the global minimum with the next lowest-lying isomer, namely AlNCCC, located more than $20 \mathrm{kcal} / \mathrm{mol}$ higher in energy. Because AlCCCN is the primary target for a possible experimental observation its geometrical parameters have been refined employing higher-level theoretical methods. In order to obtain more accurate predictions which can be useful for rotational spectroscopy, vibration-rotation interactions have been also estimated at the MP2/aug-cc-pVTZ level of theory and a correction of $-3.7 \mathrm{MHz}$ to the rotational constant has been found. After taking into account this correction the predicted rotational constant is $1329.2 \mathrm{MHz}$ or $1337.9 \mathrm{MHz}$, at the CCSD(T)/aug-cc-pVTZ level and composite method, respectively.

On this basis, wide frequency scans from 5 to $6 \mathrm{GHz}$ were directed to detect plausible signals corresponding to the $J=2-1$ rotational transitions of the linear isomers of $\mathrm{AlC}_{3} \mathrm{~N}$. A rotational transition centered around $5365 \mathrm{MHz}$, with a very complex hyperfine structure showing many fully resolved components (as shown in the upper panel of Figure 2) was finally found. The experimental value match nicely with that predicted by the composite method for the $J=2-1$ 
TABLE II. Theoretical and experimental spectroscopic constants for Al$\mathrm{CCCN}$ isomer. Theoretical rotational constants have been corrected with vibration-rotation interaction estimated at MP2/aug-cc-pVTZ level.

\begin{tabular}{lcc}
\hline \hline Parameter & Theoretical & Experimental \\
\hline $\mathrm{B}(\mathrm{MHz})$ & $1329.2^{\mathrm{a}} / 1337.9^{\mathrm{b}}$ & $1340.757500(32)$ \\
$\mathrm{D}(\mathrm{kHz})$ & $0.0600^{\mathrm{c}}$ & $0.0673(14)$ \\
$\mathrm{eQq}(\mathrm{Al})(\mathrm{MHz})$ & $-38.224^{\mathrm{d}}$ & $-38.5993(8)$ \\
$\mathrm{eQq}(\mathrm{N})(\mathrm{MHz})$ & $-4.458^{\mathrm{d}}$ & $-4.2475(6)$ \\
$\mathrm{C}_{\mathrm{I}}(\mathrm{Al})(\mathrm{kHz})$ & $\ldots$ & $1.257(31)$ \\
$\mathrm{rms}(\mathrm{kHz})$ & $\ldots$ & 0.6 \\
$\mathrm{~N}$ & $\ldots$ & 73 \\
\hline \hline
\end{tabular}

${ }^{\mathrm{a}} \mathrm{CCSD}(\mathrm{T}) /$ aug-cc-pVQZ level.

${ }^{\mathrm{b}}$ Composite method.

${ }^{\mathrm{c} M P 2 / \text { aug-cc-pVTZ. }}$

${ }^{\mathrm{d} C C S D / a u g-c c-p V T Z \text {. }}$

transition of the linear AlCCCN isomer at $5351 \mathrm{MHz}$. However, only a conclusive identification of the observed transition comes from the comparison between the predicted and observed nuclear quadrupole hyperfine structure. As can be seen in Figure 2, an exceptional matching exists between the observed and predicted spectra, when considering the eQq values for the ${ }^{27} \mathrm{Al}$ and ${ }^{14} \mathrm{~N}$ nuclei of Table II for the linear $\mathrm{AlCCCN}$ isomer.

An initial fit, ${ }^{31}$ including the 27 hyperfine components measured for the $J=2-1$ rotational transition gives rise a first set of constants which were used to predict and measure the $J=1-0, J=3-2$, and $J=4-3$ centered at 2683,8044 , and $10725 \mathrm{MHz}$, respectively. A total of 73 hyperfine components (collected in Table III) were analyzed ${ }^{31}$ using a ${ }^{1} \Sigma$ Hamiltonian of the following form: ${ }^{32} H=H_{R}+H_{Q}+H_{n s r}$ where $H_{R}$ contains rotational and centrifugal distortion parameters, $H_{Q}$ the quadrupole coupling interactions while $\mathrm{H}_{\mathrm{nsr}}$ describes the nuclear spin-rotation terms. The energy levels involved in each transition are labeled with the quantum numbers $\mathrm{J}, \mathrm{F}_{1}$, and $\mathrm{F}$, where $\mathrm{F}_{1}=\mathrm{J}+\mathrm{I}_{\mathrm{Al}}$ and $\mathrm{F}=\mathrm{F}_{1}+\mathrm{I}_{\mathrm{N}}$. The experimental values for the rotational parameters $B_{0}$ and $D$, the electric quadrupole coupling constant $e Q q$ for the ${ }^{27} \mathrm{Al}$ and ${ }^{14} \mathrm{~N}$ nuclei along with the nuclear spin-rotation parameter $C_{\mathrm{I}}\left({ }^{27} \mathrm{Al}\right)$ for the aluminum nucleus were derived from the analysis. Attempting to fit this last constant for the ${ }^{14} \mathrm{~N}$ nucleus resulted in values that were undefined to within their $3 \sigma$ uncertainties. The standard deviation obtained for the fit is $0.6 \mathrm{kHz}$. Confirmation of the observed species as the linear AlCCCN isomer resides in the excellent agreement between the experimental and theoretical values of the rotational and ${ }^{27} \mathrm{Al}$ and ${ }^{14} \mathrm{~N}$ nuclear quadrupole coupling constants collected in Table II.

Additional searches to find rotational signatures of other species of $\mathrm{AlC}_{3} \mathrm{~N}$ failed. At this point, it is interesting to note that both aluminum cyanide and isocyanide have been experimentally observed by rotational spectroscopy, ${ }^{33,34}$ being AlNC predicted to be about $5 \mathrm{kcal} / \mathrm{mol}$ more stable than AlCN. ${ }^{35,36} \mathrm{BrCCCN}$ can be considered as a good generator of Metal-CCCN species but it might not be suitable for generating M-NCCC species, which is an important issue to consider in the non-observation of AlNCCC in our experiment. In addition, in the case of $\mathrm{AlC}_{3} \mathrm{~N}$ species the cyanide isomer is
TABLE III. Measured frequencies and residuals (in MHz) for the nuclear quadrupole coupling hyperfine components of AlCCCN.

\begin{tabular}{|c|c|c|c|c|c|c|c|}
\hline $\mathbf{J}^{\prime}$ & $\mathrm{J}^{\prime \prime}$ & $\mathrm{F}_{1}^{\prime}$ & $\mathrm{F}^{\prime \prime}{ }_{1}$ & $\mathrm{~F}^{\prime}$ & $\mathrm{F}^{\prime \prime}$ & $v_{\text {obs. }}$ & $v_{\text {obs. }}-v_{\text {calc. }}$ \\
\hline \multirow[t]{9}{*}{1} & 0 & 2.5 & 2.5 & 1.5 & 1.5 & 2674.63471 & -0.00049 \\
\hline & & 2.5 & 2.5 & 3.5 & 3.5 & 2675.05122 & -0.00046 \\
\hline & & 2.5 & 2.5 & 2.5 & 2.5 & 2676.00137 & -0.00024 \\
\hline & & 3.5 & 2.5 & 3.5 & 2.5 & 2682.89096 & -0.00029 \\
\hline & & 3.5 & 2.5 & 2.5 & 1.5 & 2683.57333 & -0.00196 \\
\hline & & 3.5 & 2.5 & 4.5 & 3.5 & 2683.66385 & -0.00011 \\
\hline & & 1.5 & 2.5 & 1.5 & 1.5 & 2686.77891 & -0.00138 \\
\hline & & 1.5 & 2.5 & 0.5 & 1.5 & 2687.13311 & -0.00050 \\
\hline & & 1.5 & 2.5 & 2.5 & 3.5 & 2687.41089 & -0.00059 \\
\hline \multirow[t]{27}{*}{2} & 1 & 2.5 & 1.5 & 1.5 & 0.5 & 5354.26762 & -0.00106 \\
\hline & & 2.5 & 1.5 & 3.5 & 2.5 & 5354.35676 & 0.00020 \\
\hline & & 2.5 & 1.5 & 2.5 & 2.5 & 5354.93291 & -0.00076 \\
\hline & & 2.5 & 1.5 & 2.5 & 1.5 & 5355.56552 & -0.00025 \\
\hline & & 3.5 & 3.5 & 2.5 & 2.5 & 5356.10914 & 0.00042 \\
\hline & & 3.5 & 3.5 & 4.5 & 4.5 & 5356.20544 & 0.00001 \\
\hline & & 3.5 & 3.5 & 3.5 & 3.5 & 5356.64182 & -0.00018 \\
\hline & & 3.5 & 2.5 & 3.5 & 2.5 & 5363.53147 & -0.00017 \\
\hline & & 4.5 & 1.5 & 3.5 & 2.5 & 5360.55638 & -0.00009 \\
\hline & & 4.5 & 3.5 & 4.5 & 3.5 & 5363.65193 & 0.00049 \\
\hline & & 1.5 & 1.5 & 2.5 & 1.5 & 5360.44958 & -0.00053 \\
\hline & & 1.5 & 1.5 & 1.5 & 2.5 & 5360.50497 & -0.00007 \\
\hline & & 1.5 & 1.5 & 1.5 & 0.5 & 5360.78391 & 0.00008 \\
\hline & & 4.5 & 3.5 & 5.5 & 4.5 & 5363.95089 & 0.00037 \\
\hline & & 1.5 & 1.5 & 1.5 & 1.5 & 5361.13692 & -0.00022 \\
\hline & & 4.5 & 3.5 & 3.5 & 2.5 & 5364.39334 & -0.00023 \\
\hline & & 3.5 & 2.5 & 3.5 & 3.5 & 5364.48435 & 0.00064 \\
\hline & & 3.5 & 2.5 & 4.5 & 3.5 & 5364.81829 & 0.00058 \\
\hline & & 0.5 & 1.5 & 1.5 & 2.5 & 5364.93107 & 0.00021 \\
\hline & & 3.5 & 2.5 & 2.5 & 1.5 & 5365.04869 & -0.00012 \\
\hline & & 0.5 & 1.5 & 0.5 & 0.5 & 5365.18383 & -0.00009 \\
\hline & & 0.5 & 1.5 & 0.5 & 1.5 & 5365.53805 & 0.00081 \\
\hline & & 2.5 & 2.5 & 3.5 & 2.5 & 5365.76522 & 0.00093 \\
\hline & & 2.5 & 2.5 & 2.5 & 2.5 & 5366.34201 & 0.00061 \\
\hline & & 2.5 & 2.5 & 3.5 & 3.5 & 5366.71673 & 0.00037 \\
\hline & & 2.5 & 2.5 & 1.5 & 1.5 & 5366.76660 & -0.00049 \\
\hline & & 2.5 & 2.5 & 2.5 & 3.5 & 5367.29364 & 0.00017 \\
\hline \multirow[t]{24}{*}{3} & 2 & 4.5 & 4.5 & 5.5 & 5.5 & 8037.77458 & 0.00109 \\
\hline & & 1.5 & 0.5 & 2.5 & 1.5 & 8041.00381 & 0.00079 \\
\hline & & 2.5 & 1.5 & 2.5 & 1.5 & 8041.06585 & -0.00021 \\
\hline & & 2.5 & 1.5 & 3.5 & 2.5 & 8041.68165 & 0.00057 \\
\hline & & 3.5 & 2.5 & 3.5 & 2.5 & 8042.11209 & 0.00081 \\
\hline & & 2.5 & 1.5 & 1.5 & 0.5 & 8042.15330 & 0.00041 \\
\hline & & 4.5 & 2.5 & 3.5 & 2.5 & 8043.70899 & -0.00040 \\
\hline & & 3.5 & 2.5 & 2.5 & 1.5 & 8043.78790 & 0.00070 \\
\hline & & 3.5 & 2.5 & 4.5 & 3.5 & 8043.81635 & -0.00030 \\
\hline & & 5.5 & 4.5 & 5.5 & 5.5 & 8043.82945 & -0.00101 \\
\hline & & 4.5 & 3.5 & 4.5 & 4.5 & 8044.50608 & -0.00075 \\
\hline & & 3.5 & 3.5 & 3.5 & 2.5 & 8044.77355 & 0.00022 \\
\hline & & 4.5 & 3.5 & 4.5 & 3.5 & 8044.84035 & -0.00048 \\
\hline & & 5.5 & 4.5 & 5.5 & 4.5 & 8044.90024 & 0.00014 \\
\hline & & 5.5 & 4.5 & 4.5 & 3.5 & 8044.98067 & 0.00003 \\
\hline & & 4.5 & 3.5 & 5.5 & 4.5 & 8045.51866 & 0.00008 \\
\hline & & 1.5 & 1.5 & 1.5 & 1.5 & 8045.67326 & 0.00124 \\
\hline & & 1.5 & 1.5 & 2.5 & 2.5 & 8046.11592 & 0.00005 \\
\hline & & 4.5 & 3.5 & 3.5 & 2.5 & 8046.37197 & 0.00053 \\
\hline & & 4.5 & 3.5 & 3.5 & 3.5 & 8046.51845 & -0.00070 \\
\hline & & 2.5 & 2.5 & 2.5 & 2.5 & 8046.63682 & -0.00061 \\
\hline & & 2.5 & 2.5 & 3.5 & 3.5 & 8047.14293 & 0.00040 \\
\hline & & 2.5 & 2.5 & 1.5 & 1.5 & 8047.23619 & -0.00079 \\
\hline & & 2.5 & 3.5 & 2.5 & 3.5 & 8049.44645 & -0.00074 \\
\hline
\end{tabular}


TABLE III. (Continued.)

\begin{tabular}{|c|c|c|c|c|c|c|c|}
\hline $\mathrm{J}^{\prime}$ & $\mathrm{J}^{\prime \prime}$ & $\mathrm{F}_{1}^{\prime}$ & $\mathrm{F}^{\prime \prime}{ }_{1}$ & $\mathrm{~F}^{\prime}$ & $\mathrm{F}^{\prime \prime}$ & $v_{\text {obs. }}$ & $v_{\text {obs. }}-v_{\text {calc. }}$ \\
\hline \multirow[t]{13}{*}{4} & 3 & 2.5 & 1.5 & 2.5 & 1.5 & 10724.05291 & -0.00040 \\
\hline & & 3.5 & 2.5 & 3.5 & 2.5 & 10724.49903 & 0.00018 \\
\hline & & 2.5 & 1.5 & 3.5 & 2.5 & 10724.69515 & -0.00129 \\
\hline & & 3.5 & 2.5 & 4.5 & 3.5 & 10724.98315 & 0.00030 \\
\hline & & 1.5 & 0.5 & 2.5 & 1.5 & 10725.34913 & 0.00028 \\
\hline & & 4.5 & 3.5 & 4.5 & 3.5 & 10725.73774 & -0.00044 \\
\hline & & 1.5 & 0.5 & 0.5 & 0.5 & 10725.74059 & -0.00014 \\
\hline & & 4.5 & 3.5 & 3.5 & 2.5 & 10725.88506 & -0.00013 \\
\hline & & 4.5 & 3.5 & 5.5 & 4.5 & 10726.00496 & 0.00018 \\
\hline & & 4.5 & 4.5 & 5.5 & 5.5 & 10726.20217 & 0.00066 \\
\hline & & 5.5 & 4.5 & 5.5 & 4.5 & 10726.25886 & -0.00028 \\
\hline & & 6.5 & 5.5 & 5.5 & 4.5 & 10726.32945 & 0.00095 \\
\hline & & 5.5 & 4.5 & 4.5 & 3.5 & 10726.37781 & -0.00059 \\
\hline
\end{tabular}

$20 \mathrm{kcal} / \mathrm{mol}$ more stable than the isocyanide isomer. Hence, although AlNCCC was actually generated in our experiment it would not be enough populated to be detected.

The electronic quadrupole coupling constant for the $\mathrm{Al}$ nucleus was found to be eQq $(\mathrm{Al})=-38.5993$ (8) $\mathrm{MHz}$. This value can be compared with those for other aluminum containing species to gain some insights about the bonding properties of Al-C union. The quadrupole constant of AlCCCN lies between those for AlCCH $(-42.3917(65) \mathrm{MHz})^{21}$ and AlCN (-37.2225 (29) MHz). ${ }^{33}$ These numbers suggest that the bond Al-C in AlCCCN is not as covalent as AlCCH and presents some ionic character like in the AlCN system. This fact is reflected in the Al-C bond lengths, shown in Table IV. The previous conclusion has been confirmed by using a topological analysis of the electronic density in the framework of the Bader's Quantum Theory of Atoms in Molecules (QTAIM). ${ }^{38}$ This analysis was performed for each stationary point on the PES's using the Keith's AIMAll package ${ }^{39}$ including standard thresholds. Results for AlCN, AlCCCN, and $\mathrm{AlCCH}$ are shown in Table $\mathrm{V}$ and the corresponding contour maps of the Laplacian of the electron density are shown in Figure S1 of the supplementary material. ${ }^{45}$ In this context two limiting types of interactions can be identified namely shared and closed-shells interactions. ${ }^{40}$ In shared interactions, typical of covalent compounds, the nuclei are bound as a consequence of the lowering of the potential energy associated with the concentration of electronic charge shared between the nuclei; this is reflected in relatively large values of $\rho(\mathrm{r})$ and negative values of the Laplacian, $\nabla^{2} \rho(\mathrm{r})$ at the critical point. The

TABLE IV. Nuclear quadrupole coupling constant for the Al nucleus and Al-C bond distances for some Al-containing species.

\begin{tabular}{llc}
\hline \hline Species & eQq $(\mathrm{Al})(\mathrm{MHz})$ & Al-C $(\AA)$ \\
\hline AlCCH & $-42.3917(65)^{\mathrm{a}}$ & $1.986^{\mathrm{b}}$ \\
$\mathrm{AlCCCN}$ & $-38.5993(8)^{\mathrm{c}}$ & $2.008^{\mathrm{d}}$ \\
$\mathrm{AlCN}$ & $-37.2225(29)^{\mathrm{e}}$ & $2.015^{\mathrm{f}}$ \\
\hline \hline
\end{tabular}

${ }^{\mathrm{a}}$ Reference 21 .

${ }^{\mathrm{b}}$ Reference 37

${ }^{\mathrm{c}}$ This work.

${ }^{\mathrm{d}}$ Theoretical value of this work.

${ }^{\mathrm{e}}$ Reference 33 .

${ }^{\mathrm{f}}$ Theoretical value from Ref. 35 .
TABLE V. Local topological properties (in au.) of the electronic charge density distribution calculated at the position of the bond critical points for the different (AIMAll) species. ${ }^{\text {a }}$

\begin{tabular}{lllccc}
\hline \hline Species & Bond & $\rho(\mathrm{r})$ & $\nabla^{2} \rho(\mathrm{r})$ & $|\mathrm{V}(\mathrm{r})| \mathrm{G}(\mathrm{r})$ & $H(\mathrm{r})$ \\
\hline \multirow{2}{*}{ AlCN } & $\mathrm{Al}-\mathrm{C}$ & 0.069 & 0.242 & 1.214 & -0.016 \\
& $\mathrm{C}-\mathrm{N}$ & 0.487 & -0.457 & 2.142 & -0.919 \\
\multirow{2}{*}{ AlCCCN } & $\mathrm{Al}-\mathrm{C}_{1}$ & 0.072 & 0.266 & 1.205 & -0.017 \\
& $\mathrm{C}_{1}-\mathrm{C}_{2}$ & 0.413 & -1.357 & 3.018 & -0.672 \\
& $\mathrm{C}_{2}-\mathrm{C}_{3}$ & 0.313 & -1.043 & 4.488 & -0.366 \\
& $\mathrm{C}_{3}-\mathrm{N}$ & 0.482 & -0.333 & 2.102 & -0.898 \\
\multirow{2}{*}{$\mathrm{AlCCH}$} & $\mathrm{Al}_{-}-\mathrm{C}_{1}$ & 0.075 & 0.289 & 1.204 & -0.019 \\
& $\mathrm{C}_{1}-\mathrm{C}_{2}$ & 0.410 & -1.350 & 3.058 & -0.657 \\
& $\mathrm{C}_{2}-\mathrm{H}$ & 0.299 & -1.253 & 10.458 & -0.350 \\
\hline \hline
\end{tabular}

${ }^{\text {a }}$ The electronic charge density $[\rho(\mathrm{r})]$, the Laplacian $\left[\nabla^{2} \rho(\mathrm{r})\right]$, the relationship between the potential energy density V(r) and the lagrangian form of kinetic energy density G(r)) and the total energy density, $[H(\mathrm{r})]$

second limiting type of atomic interaction is that occurring between closed-shell systems, such as those found in ionic bond or van der Waals molecules for instance. In these interactions, $\rho(\mathrm{r})$ is relatively low in value and the Laplacian, $\nabla^{2} \rho(\mathrm{r})$, is positive.

Another useful property to characterize the degree of covalence of a bond is the total energy density $H(\mathrm{r})$. It is defined as the sum of the potential energy density, $V(\mathrm{r})$ and the gradient kinetic energy density $G(r)$ at a critical point. In covalent interactions $H(\mathrm{r})$ is negative in value ${ }^{41}$ and the positive value of $H(\mathrm{r})$ is characteristic of ionic interactions and van der Waals systems. ${ }^{40}$ The covalent character of an interaction can also be quantitatively analyzed by taking into account the $|V(\mathrm{r})| / G(\mathrm{r})$ ratio. In covalent interactions the value of this relationship is greater than 2. It is smaller than 1 for non-covalent interactions and between 1 and 2 for partially covalent bonds.

The local topological properties of the carbon-carbon and carbon-nitrogen bond critical points are indicative of shared interactions: large values of electron density, negative values of its Laplacian, $|V(\mathrm{r})| / G(\mathrm{r}) \mid$ ratios greater than 2 and negative values of the total energy densities $H(\mathrm{r})$. On the opposite, the aluminum-carbon bond critical points show low values of $\rho(\mathrm{r})$ and positive values of its Laplacian. The $|V(\mathrm{r})| / G(\mathrm{r}) \mid$ ratios are between 1 and 2 and $H(\mathrm{r})$ is negative with a low value. Thus these interactions can be classified as closed shell interactions (typical of ionic compounds) with a small degree of covalence.

On the other hand, the quasi identical eQq values for ${ }^{14} \mathrm{~N}$ nucleus in AlCCCN and $\mathrm{HCCCN},-4.2475$ (6) $\mathrm{MHz}$ and -4.31924 (1) $\mathrm{MHz}^{42}$ respectively, indicate that the electronic environment around the ${ }^{14} \mathrm{~N}$ nucleus is very similar in both species. Thus, one can infer that the nature of the bond in the $\mathrm{C} \equiv \mathrm{N}$ group in AlCCCN should be the same to that found in $\mathrm{HCCCN}$.

Finally, the rotational constants derived from the observed laboratory spectrum of AlCCCN allow performing a search in the millimeter domain using the $3 \mathrm{~mm}$ line survey of IRC +10216 taken with the 30-m IRAM radiotelescope. ${ }^{43,44}$ The values of the experimental constants obtained in the present investigation have been used to predict its transitions 
in the $3 \mathrm{~mm}$ domain with uncertainties of $0.15-0.4 \mathrm{MHz}$, which is enough to search for lines having total frequency coverage in IRC +10216 of $\sim 10 \mathrm{MHz}$ at these frequencies (total linewidth $\sim 29 \mathrm{~km} / \mathrm{s}$ ). Unfortunately, we have not detected any of the lines covered in the line survey, from $\mathrm{J}=30-29$ up to $\mathrm{J}=43-42$. From the most sensitive spectra we obtain an upper limit $(3 \sigma)$ to the column density of AlC$\mathrm{CCN}$ in front of IRC +10216 of $<3 \times 10^{12} \mathrm{~cm}^{-2}$. Note that, AlNC has been detected ${ }^{6}$ in this source with a column density of $9 \times 10^{11} \mathrm{~cm}^{-2}$. The upper limit obtained for the column density of AlCCCN is 3 times higher than that of AlNC. Although the dipole moment of AlCCCN is higher than that of AlNC, its partition function is much higher and it could be much better to search for this species in the $7 \mathrm{~mm}$ domain.

\section{CONCLUSIONS}

The first metal cyanoacetylide, AlCCCN, has been produced and characterized in the laboratory using a combination of laser ablation techniques and Fourier transform microwave spectroscopy. A newly constructed ablation-heating source has been proved as an effective method to create metallic cyanoacetylides, using solid samples of BrCCCN as main precursor.

The predictions for the rotational constant at both CCSD(T)/aug-cc-pVQZ and composite methods are in good agreement with the experimental value. Since the composite method is more affordable in terms of computational demands than the CCSD(T)/aug-cc-pVQZ level, our results suggest that the composite method corrected with vibration-rotation interaction can provide good estimates of rotational constants for this type of compounds.

We have searched for AlCCCN species towards the carbon-rich evolved star IRC + 10216 and obtained only upper limits. Important information on the chemistry of metal cyanides and isocyanides could be obtained if M-CCCN species are detected in the circumstellar medium. $\mathrm{Mg}$ - and $\mathrm{Na}-\mathrm{C}_{3} \mathrm{~N}$ are the most obvious candidates after $\mathrm{AlC}_{3} \mathrm{~N}$ and will be studied soon in our laboratory. Although their detection seems to be difficult within the state-of-the-art observations of evolved stars, the sensitivity that will be provided by the ALMA interferometer in the next years could open the possibility to detect these species in circumstellar envelopes.

\section{ACKNOWLEDGMENTS}

This research has been supported by the Ministerio de Ciencia e Innovación of Spain (Grants CTQ2010-19008, Consolider-Ingenio 2010 CSD2009-00038, and CTQ201016864), by European Research Council under the European Union's Seventh Framework Programme (FP/ 2007-2013)/ERC-2013-SyG (Grant Agreement No. 610256 NANOCOSMOS) and by the Junta de Castilla y León (Grants VA175U13 and VA077U13). C.C. thanks the Junta de Castilla y León for a postdoctoral contract (Grant CIP13/01). J.C.G. thanks the Centre National d'Etudes Spatiales (CNES) and the Program PCMI (INSU-CNRS) for financial support. J.-C.G. and J.C. received the support of the contract
ANR-13-BS05-0008 IMOLABS. We thank Marcelino Agúndez for useful comments and suggestions.

${ }^{1}$ J. Cernicharo and M. Guélin, Astron. Astrophys. 183, L10 (1987).

${ }^{2}$ M. Agúndez, J. P. Fonfría, J. Cernicharo, C. Kahane, F. Daniel, and M. Guelin, Astron. Astrophys. 543, A48 (2012).

${ }^{3}$ L. M. Ziurys, A. J. Apponi, and T. G. Phillips, Astrophys. J. 433, 729 (1994).

${ }^{4}$ E. D. Tenenbaum and L. M. Ziurys, Astrophys. J. 694, L59 (2009).

${ }^{5}$ E. D. Tenenbaum and L. M. Ziurys, Astrophys. J. 712, L93 (2010).

${ }^{6}$ L. M. Ziurys, C. Savage, J. L. Highberger, A. J. Apponi, M. Guélin, and J. Cernicharo, Astrophys. J. 564, L45 (2002).

${ }^{7}$ B. E. Turner, T. C. Steimle, and L. Meerts, Astrophys. J. 426, 97 (1994)

${ }^{8}$ R. L. Pulliam, C. Savage, M. Agúndez, J. Cernicharo, M. Guélin, and L. M. Ziurys, Astrophys. J. 725, L181 (2010).

${ }^{9}$ K. Kawaguchi, E. Kagi, T. Hirano, S. Takano, and S. Saito, Astrophys. J. 406, L39 (1993).

${ }^{10}$ L. M. Ziurys, A. J. Apponi, M. Guélin, and J. Cernicharo, Astrophys. J. 445, L47 (1995).

${ }^{11}$ L. N. Zack, D. T. Halfen, and L. M. Ziurys, Astrophys. J. 733, L36 (2011).

${ }^{12}$ M. Guélin, S. Muller, J. Cernicharo, A. J. Apponi, M. C. McCarthy, C. A. Gottlieb, and P. Thaddeus, Astron. Astrophys. 363, L9 (2000).

${ }^{13}$ M. Guélin, S. Muller, J. Cernicharo, M. C. McCarthy, and P. Thaddeus, Astron. Astrophys. 426, L49 (2004).

${ }^{14}$ C. Cabezas, J. Cernicharo, J. L. Alonso, M. Agúndez, S. Mata, M. Guélin, and I. Peña, Astrophys. J. 775, 133 (2013)

${ }^{15}$ M. B. Bell, P. A. Feldman, M. J. Travers, M. C. McCarthy, C. A. Gottlieb, and P. Thaddeus, Astrophys. J. 483, L61 (1997).

${ }^{16}$ E. Herbst, Chem. Soc. Rev. 30, 168 (2001).

${ }^{17}$ J. L. Alonso, C. Pérez, M. E. Sanz, J. C. López, and S. Blanco, Phys. Chem. Chem. Phys. 11, 617 (2009) and references therein.

${ }^{18}$ C. Bermúdez, S. Mata, C. Cabezas, and J. L. Alonso, Angew. Chem., Int. Ed. 53, 6655 (2014).

${ }^{19}$ M. E. Sanz, C. Cabezas, S. Mata, and J. L. Alonso, J. Chem. Phys. 140, 204308 (2014)

${ }^{20}$ E. Kloster-Jensen, Acta Chem. Scand. 17, 1862-1865 (1963).

${ }^{21}$ C. Cabezas, S. Mata, A. M. Daly, A. Martín, J. L. Alonso, and J. Cernicharo, J. Mol. Spectrosc. 278, 31 (2012).

${ }^{22}$ S. Petrie, Mon. Not. R. Astron. Soc. 302, 482 (1999).

${ }^{23}$ C. MØller and M. Plesset, Phys. Rev. 46, 618 (1934).

${ }^{24}$ T. H. Dunning, J. Chem. Phys. 90, 1007 (1989).

${ }^{25}$ K. Raghavachari, G. W. Trucks, J. A. Pople, and M. Head-Gordon, Chem. Phys. Lett. 157, 479 (1989).

${ }^{26}$ M. Heckert, M. Kalay, and J. Gauss, Mol. Phys. 103, 2109 (2005).

${ }^{27}$ M. Heckert, M. Kalay, D. P. Tew, W. Klopper, and J. Gauss, J. Chem. Phys. 125, 044108 (2006).

${ }^{28}$ V. Barone, M. Biczysko, J. Bloino, and C. Puzzarini, J. Chem. Theory Comput. 9, 1533 (2013).

${ }^{29}$ M. J. Frisch, G. W. Trucks, H. B. Schlegel et al. Gaussian 09, Revision B.01, Gaussian, Inc., Wallingford, CT, 2010

${ }^{30}$ CFOUR, a quantum chemical program package written by J. F. Stanton, J. Gauss, M. E. Harding, and P. G. Szalay with contributions from A. A. Auer, R. J. Bartlett, U. Benedikt, C. Berger, D. E. Bernholdt, Y. J. Bomble, L. Cheng, O. Christiansen, M. Heckert, O. Heun, C. Huber, T.-C. Jagau, D. Jonsson, J. Jusélius, K. Klein, W. J. Lauderdale, D. A. Matthews, T. Metzroth, L. A. Mück, D. P. O'Neill, D. R. Price, E. Prochnow, C. Puzzarini, K. Ruud, F. Schiffmann, W. Schwalbach, C. Simmons, S. Stopkowicz, A. Tajti, J. Vázquez, F. Wang, J. D. Watts and the integral packages MOLECULE (J. Almlöf and P. R. Taylor), PROPS (P. R. Taylor), ABACUS (T. Helgaker, H. J. Aa. Jensen, P. Jørgensen, and J. Olsen), and ECP routines by A. V. Mitin and C. van Wüllen, 2013.

${ }^{31}$ H. M. Pickett, J. Mol. Spectrosc. 148, 371 (1991).

${ }^{32}$ W. Gordy and R. L. Cook, Microwave Molecular Spectra, 3rd ed. (Wiley, New York, 1984).

${ }^{33}$ K. A. Walker and M. C. L. Gerry, Chem. Phys. Lett. 301, 200 (1999).

${ }^{34}$ J. S. Robinson, A. J. Apponi, and L. M. Ziurys, Chem. Phys. Lett. 278, 1 (1997).

${ }^{35}$ B. Ma, Y. Yamaguchi, and H. F. Schaefer III, Mol. Phys. 86, 1331 (1995).

${ }^{36}$ S. Petrie, J. Phys. Chem. 100, 11581 (1996).

${ }^{37}$ M. Sun, D. T. Halfen, D. J. Clouthier, and L. M. Ziurys, Chem. Phys. Lett. 553, 11-16 (2012).

${ }^{38}$ R. W. F. Bader, Atoms in Molecules: A Quantum Theory (Clarendon Press, New York, 1990). 
${ }^{39}$ T. A. Keith, AIMAll, version 13.11.04, Professional, TK Gristmill Software: Overland Park, KS, 2013, see http://aim.tkgristmill.com.

${ }^{40}$ R. W. F. Bader, Chem. Rev. 91, 893-928 (1991).

${ }^{41}$ D. Cremer and E. Kraka, Angew. Chem., Int. Ed. 23, 627-628 (1984).

${ }^{42}$ R. L. Deleonb and J. S. Muenter, J. Chem. Phys. 82, 1702 (1985).

${ }^{43}$ J. Cernicharo, M. Guélin, M. Agúndez, M. C. McCarthy, and P. Thaddeus, Astrophys. J. 688, L83-L86 (2008).
${ }^{44}$ Cernicharo et al. "A sensitive line survey at $3 \mathrm{~mm}$ with the $30 \mathrm{~m}$ IRAM radio telescope of IRC+10216" (unpublished).

${ }^{45}$ See supplementary material at http://dx.doi.org/10.1063/1.4894501 for Table S1, with the anharmonic vibrational frequencies and IR intensities for AlCCCN evaluated at the MP2/aug-cc-pVTZ level and Figure S1, with the contour maps of the Laplacian distribution of the electron density for the $\mathrm{AlCN}, \mathrm{AlCCCN}$, and $\mathrm{AlCCH}$ species. 